\title{
SYMPLECTIC CUTTING OF KÄHLER MANIFOLDS
}

\author{
MAXIM BRAVERMAN
}

\begin{abstract}
We obtain estimates on the character of the cohomology of an $S^{1}$-equivariant holomorphic vector bundle over a Kähler manifold $M$ in terms of the cohomology of the Lerman symplectic cuts and the symplectic reduction of $M$. In particular, we prove and extend inequalities conjectured by Wu and Zhang [12].

The proof is based on constructing a flat family of complex spaces $M_{t}(t \in \mathbb{C})$ such that $M_{t}$ is isomorphic to $M$ for $t \neq 0$, while $M_{0}$ is a singular reducible complex space, whose irreducible components are the Lerman symplectic cuts.
\end{abstract}

\section{INTRODUCTION}

Let $M$ be a smooth Kähler manifold of complex dimension $n$ endowed with a holomorphic Hamiltonian action of the circle group $S^{1}$. Let $\mu: M \rightarrow \mathbb{R}$ denote the moment map for this action. Assume that 0 is a regular value of $\mu$ and that $S^{1}$ acts freely on $\mu^{-1}(0)$. Using a construction of E. Lerman, [7], one can "cut" $M$ into two smooth Kähler manifolds $M_{+}$and $M_{-}$endowed with a holomorphic circle action. The symplectic reduction $M_{\text {red }}=\mu^{-1}(0) / S^{1}$ of $M$ is embedded into $M_{ \pm}$as a connected component of the fixed point set.

Let $E$ be an equivariant holomorphic vector bundle over $M$. Then $E$ induces a holomorphic vector bundle $E_{\text {red }}$ over $M_{\text {red }}$ and equivariant holomorphic bundles $E_{ \pm}$over $M_{ \pm}$. In this paper we show that there are Morse-type inequalities which estimate the character of the $S^{1}$-action on the cohomology $H^{*}(M, \mathcal{O}(E))$ of the sheaf of holomorphic sections of $E$ in terms of the cohomology $H^{*}\left(M_{ \pm}, \mathcal{O}\left(E_{ \pm}\right)\right), H^{*}\left(M_{\text {red }}, \mathcal{O}\left(E_{\text {red }}\right)\right)$ of the sheaves of holomorphic sections of the bundles $E_{ \pm}$and $E_{\text {red }}$ respectively. These inequalities were conjectured by $\mathrm{Wu}$ and Zhang [12] for the case when $E$ is a pre-quantum line bundle.

As a consequence, we obtain a new geometric proof of the "gluing formula" for the index of $E$ ([3], 9], see (2.2)) for the case when the manifold $M$ is Kähler.

Our proof is based on the following geometric construction which, we believe, is interesting by itself. We consider the union $M_{\text {cut }}$ of $M_{ \pm}$along $M_{\text {red }}$. Thus $M_{\text {cut }}$ is a singular reducible complex space, whose smooth irreducible components $M_{ \pm}$intersect by the symplectic reduction $M_{\text {red }}$. We show that $M_{\text {cut }}$ may be considered as a deformation of $M$.

This research was partially supported by grant No. 96-00210/1 from the United States-Israel Binational Science Foundation (BSF), Jerusalem, Israel. 
More precisely, we construct a family $M_{t}$ of complex spaces parameterized by a complex parameter $t$, such that $M_{t}$ is complex isomorphic to $M$ for any $t \neq 0$ while $M_{0}$ is complex isomorphic to $M_{c u t}$. It turns out that $M_{t}$ is a flat family of complex spaces. That implies that the dimension (and also the character) of the cohomology of $M_{t}$ is an upper semi-continuous function of $t$. In particular, the character of $H^{*}\left(M_{\text {cut }}, \mathcal{O}\left(E_{\text {cut }}\right)\right)$ is greater than the character of $H^{*}(M, \mathcal{O}(E)$ ) (a partial order on the ring of characters is introduced in Definition 2.6). Moreover, there are Morse-type inequalities (cf. Theorem 2.7) which estimate the character of $H^{*}(M, \mathcal{O}(E))$ in terms of the character of $H^{*}\left(M_{\text {cut }}, \mathcal{O}\left(E_{\text {cut }}\right)\right)$.

The cohomology $H^{*}\left(M_{\text {cut }}, \mathcal{O}\left(E_{\text {cut }}\right)\right)$ of the space $M_{\text {cut }}$ can be, in turn, calculated by means of a Mayer-Vietoris-type long exact sequence via the cohomology of $M_{ \pm}$and $M_{\text {red }}$ (cf. Section 2.9). That leads to estimates for the cohomology of $M$ in terms of the cohomology of $M_{ \pm}$and $M_{\text {red }}$.

The paper is organized as follows. In Section 2, we formulate our main results. In Section 3, we present our geometric construction of the family of complex spaces and

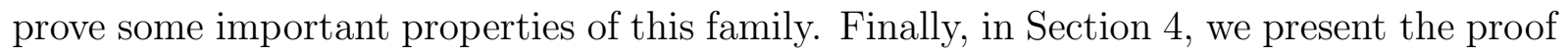
of Theorem 2.7.

Acknowledgments. I would like to thank I. Zakharevich for very useful and inspiring discussions. It was I. Zakharevich who suggested to consider the union of the Lerman symplectic cuts $M_{ \pm}$as a singular complex space.

I would like to thank the University of Warwick, where this work was completed, for hospitality.

\section{MAin RESULtS}

In this section we formulate the main results of the paper. All these results are consequences of Theorem 2.7, which will be proved in Section 4 .

2.1. Weights and formal characters. Irreducible representation of the circle group $S^{1}=\left\{e^{i \theta}: \theta \in \mathbb{R}\right\}$ are classified by integer weights (here we use the identification of the Lie algebra of $S^{1}$ with $\mathbb{R}$ which takes the negative primitive lattice element, $-2 \pi i \in i \mathbb{R}=$ $\operatorname{Lie}\left(S^{1}\right)$, to 1$)$. A representation of weight $k \in \mathbb{Z}$ is isomorphic to the complex line $\mathbb{C}$ on which the element $e^{i \theta} \in S^{1}$ acts by multiplications by $e^{-i k \theta}$.

If $W$ is a finite dimensional representation of $S^{1}$ we denote by $\operatorname{mult}_{k}(W)$ the multiplicity of the weight $k \in \mathbb{Z}$ in $W$.

The formal character of $W$ is the formal sum

$$
\operatorname{char}(W)=\sum_{k \in \mathbb{Z}} \operatorname{mult}_{k}(W) e^{-i k \theta}
$$


It lies in the ring $\mathcal{L}=\mathbb{Z}\left[e^{i \theta}, e^{-i \theta}\right]$ of Laurent polynomials in $e^{i \theta}$ with integer coefficients. This ring is called the ring of formal characters of the circle group.

2.2. Momentum map and symplectic reduction. Let $V$ denote the vector field on $M$ that generates the $S^{1}$-action and let $\omega$ denote the Kähler form on $M$. We will assume that $S^{1}$-action is Hamiltonian, i.e. there is a moment map $\mu: M \rightarrow \mathbb{R}$ such that $\iota_{V} \omega=d \mu$. Note ([四) that it is always the case if the fixed-point set of $S^{1}$ on $M$ is non-empty.

Assume that $0 \in \mathbb{R}$ is a regular value of the moment map $\mu$. Then $\mu^{-1}(0) \subset M$ is a smooth submanifold endowed with a locally free action of $S^{1}$. We will assume that this action is free. Then the quotient space $M_{r e d}=\mu^{-1}(0) / S^{1}$ is a smooth symplectic manifold called the symplectic reduction of $M$ at level 0 . The symplectic form $\omega_{\text {red }}$ on $M_{\text {red }}$ is defined by the condition that its lift on $\mu^{-1}(0)$ coincides with the restriction of $\omega$ on $\mu^{-1}(0)$.

Recall now that our manifold $M$ is Kähler and that the Kähler structure on $M$ is preserved by the circle action. In this case, the $S^{1}$ action can be canonically extended to a holomorphic action of the group of nonzero complex numbers $\mathbb{C}^{*}$ (cf. [6, Lemma 3.3]). The set

$$
M_{s}=\left\{z \cdot x: z \in \mathbb{C}^{*}, x \in \mu^{-1}(0) \subset M\right\},
$$

called the set of stable points for the $\mathbb{C}^{*}$ action, is an open submanifold of $M$, [6, Lemma 4.5], and the $\mathbb{C}^{*}$ action on $M_{s}$ is free. Obviously, the quotient of $M_{s}$ by this action is diffeomorphic to the reduced space:

$$
M_{\text {red }} \cong M_{s} / \mathbb{C}^{*}
$$

The equation (2.1), defines a canonical complex structure on $M_{r e d}$. This structure is, in fact, Kähler, and the corresponding Kähler form coincides with the form $\omega_{\text {red }}$ defined above.

Let now $E$ be a holomorphic vector bundle over $M$ which is equivariant for the $S^{1}$ action. Then the $\mathbb{C}^{*}$ action on $M$ can be also lifted on $E$. There is a unique holomorphic vector bundle $E_{\text {red }}$ over $M_{\text {red }}$ such that its pullback under the projection $M_{s} \rightarrow M_{\text {red }}$ is isomorphic to the restriction of $E$ on $M_{s}$.

2.3. Symplecting cuttings. We now recall the Lerman symplectic cutting construction, [7]. Let $\mathbb{C}_{ \pm}$denote the complex one-dimensional representations of the circle group of weights \pm 1 respectively. We endow both $\mathbb{C}_{+}$and $\mathbb{C}_{-}$with the standard Kähler form $\omega=$ $\frac{i}{2} d z \wedge d \bar{z}$. The diagonal actions of $S^{1}$ on $M \times \mathbb{C}_{ \pm}$are Hamiltonian and the corresponding moment maps are $\mu \mp \frac{1}{2}|z|^{2}$. One checks easily that 0 is a regular value for each one of these moment maps. Let us denote by $M_{ \pm}$the symplectic quotients of $M \times \mathbb{C}_{ \pm}$at level 0. The action of $S^{1}$ on the first factor of $M \times \mathbb{C}_{ \pm}$reduces to a Hamiltonian action on $M_{ \pm}$. Thus, $\left(M_{ \pm}, \omega_{ \pm}\right)$are smooth symplectic manifolds with Hamiltonian $S^{1}$-actions. 
The reduced space $M_{\text {red }}$ is embedded into $M_{ \pm}$as one of the connected components (still denoted by $M_{r e d}$ ) of the fixed points set; the compliments $M_{ \pm} \backslash M_{\text {red }}$ are $S^{1}$-equivariantly symplectomorphic to $\mu^{-1}\left(\mathbb{R}^{ \pm}\right) \subset M$, respectively. We refer to $M_{ \pm}$as symplectic cuts of $M$.

The pull-back of the bundle $E$ under the natural projection $M \times \mathbb{C}_{ \pm} \rightarrow M$ is an equivariant vector bundle over $M \times \mathbb{C}_{ \pm}$. Hence (cf. Section 2.2), it induces holomorphic vector bundles $E_{ \pm}$over $M_{ \pm}$. One of the most important facts about the cohomology of the symplectic cuts is the gluing formula (cf. [3, 9])

$$
\begin{aligned}
\sum_{p=0}^{n}(-1)^{p} & \operatorname{char} H^{p}(M, \mathcal{O}(E))=\sum_{p=0}^{n}(-1)^{p} \operatorname{char} H^{p}\left(M_{+}, \mathcal{O}\left(E_{+}\right)\right) \\
& +\sum_{p=0}^{n}(-1)^{p} \operatorname{char} H^{p}\left(M_{-}, \mathcal{O}\left(E_{-}\right)\right)-\sum_{p=0}^{n-1}(-1)^{p} \operatorname{dim}_{\mathbb{C}} H^{p}\left(M_{\text {red }}, \mathcal{O}\left(E_{\text {red }}\right)\right) .
\end{aligned}
$$

Remark 2.4. Though the individual cohomology $H^{p}(M, \mathcal{O}(E))$ has sense only for complex manifold $M$, the alternating sums which appear in (2.2) may be defined in the case when $M$ is an almost complex manifold. The formula (2.2) remains true for this more general case [3, 9] (see also [2] were the gluing formula is obtained in a still more general situation).

Let us return to the situation when $M$ is Kähler. The aim of this paper is to strengthen the gluing formula (2.2) in order to obtain an information about individual cohomology $H^{p}(M, \mathcal{O}(E))$ of $M$ in terms of the cohomology of $M_{ \pm}$and $M_{\text {red }}$.

2.5. Symplectig cutting as a singular space. Both manifolds $M_{ \pm}$contain the symplectic reduction $M_{\text {red }}$ as a submanifold. Consider the union

$$
M_{c u t}=M_{+} \cup_{M_{\text {red }}} M_{-}
$$

along $M_{\text {red }}$. Then $M_{c u t}$ is a singular reducible complex space whose irreducible components are $M_{ \pm}$and whose only singularities are the "double points" in $M_{\text {red }}$. Let $E_{\text {cut }}$ denote the vector bundle over $M_{c u t}$ whose restriction onto $M_{ \pm}$is equal to $E_{ \pm}$. The advantage of considering the singular space $M_{\text {cut }}$ rather then two disconnected manifolds $M_{ \pm}$is that $M_{\text {cut }}$ may be considered as a deformation of $M$ (cf. Section 3). This implies (cf. Theorem 2.7) estimates on char $H^{*}(M, \mathcal{O}(E)$ ) in terms of the character char $H^{*}\left(M_{\text {cut }}, \mathcal{O}\left(E_{\text {cut }}\right)\right)$ of the cohomology of the sheaf of holomorphic sections of $E_{\text {cut }}$. The cohomology $H^{*}\left(M_{\text {cut }}, \mathcal{O}\left(E_{\text {cut }}\right)\right)$ my be, in turn, calculated in terms of the cohomology of the sheaves $E_{ \pm}$and $E_{\text {red }}$ (cf. Section 2.9 bellow). That gives an estimate on char $H^{*}(M, \mathcal{O}(E))$ in terms of the spaces $M_{ \pm}, M_{\text {red }}$.

To formulate the result we need the following 
Definition 2.6. Let $q(\theta)=\sum_{k \in \mathbb{Z}} q_{k} e^{-i k \theta} \in \mathcal{L}$ be a formal character of $S^{1}$, we say $q(\theta) \geq 0$ if $q_{k} \geq 0$ for all $k \in \mathbb{Z}$. For two characters $p, q \in \mathcal{L}$, we say that $p \geq q$ if $p-q \geq 0$.

Let $Q(\theta, t)=\sum_{m=0}^{n} q_{m}(\theta) t^{m} \in \mathcal{L}[t]$ be a polynomial of degree $n$ with coefficients in $\mathcal{L}$, we say $Q(\theta, t) \geq 0$ if $q_{m}(\theta) \geq 0$ for all $m$.

Our first result is the following Morse-type inequalities between the cohomology of $M$ and $M_{c u t}$.

Theorem 2.7. There exists a polynomial $Q(\theta, t) \in \mathcal{L}[t]$, such that $Q \geq 0$ and

$$
\sum_{p=0}^{n} t^{p} \operatorname{char} H^{p}\left(M_{\text {cut }}, \mathcal{O}\left(E_{\text {cut }}\right)\right)=\sum_{p=0}^{n} t^{p} \operatorname{char} H^{p}(M, \mathcal{O}(E))+(1+t) Q(\theta, t) .
$$

Theorem 2.7 is proven in Section 4.

Remark 2.8. 1. The Morse-type inequalities (2.3) imply

$$
\operatorname{char} H^{p}\left(M_{\text {cut }}, \mathcal{O}\left(E_{\text {cut }}\right)\right) \geq \operatorname{char} H^{p}(M, \mathcal{O}(E)) \text { for any } p=0, \ldots, n .
$$

2. Substituting $t=-1$ into (2.3) we obtain the following index formula

$$
\sum_{p=0}^{n}(-1)^{p} \operatorname{char} H^{p}(M, \mathcal{O}(E))=\sum_{p=0}^{n}(-1)^{p} \operatorname{char} H^{p}\left(M_{\text {cut }}, \mathcal{O}\left(E_{\text {cut }}\right)\right) .
$$

2.9. Cohomology of $M_{\text {cut }}$. To calculate the cohomology of $M_{\text {cut }}$ with coefficients in $\mathcal{O}\left(E_{\text {cut }}\right)$ consider the equivariant short exact sequence of sheaves

$$
0 \rightarrow \mathcal{O}\left(E_{\text {cut }}\right) \stackrel{\alpha}{\longrightarrow} \mathcal{O}\left(E_{+}\right) \oplus \mathcal{O}\left(E_{-}\right) \stackrel{\beta}{\longrightarrow} \mathcal{O}\left(M_{\text {red }}\right) \rightarrow 0,
$$

Here the map $\alpha$ sends the section $s$ of $\mathcal{O}\left(E_{\text {cut }}\right)$ to the pair $\left(\left.s\right|_{M_{+}},\left.s\right|_{M_{-}}\right)$and the map $\beta$ sends the pair of sections $\left(s_{+}, s_{-}\right) \in \mathcal{O}\left(E_{+}\right) \oplus \mathcal{O}\left(E_{-}\right)$to the section $\left.s_{+}\right|_{M_{\text {red }}}-\left.s_{-}\right|_{M_{\text {red }}} \in$ $\mathcal{O}\left(M_{\text {red }}\right)$.

By standard arguments, the above short sequence leads to an equivariant long exact sequence in cohomology

$$
\begin{aligned}
\cdots \rightarrow H^{p}\left(M_{\text {cut }}, \mathcal{O}\left(E_{\text {cut }}\right)\right) \rightarrow & H^{p}\left(M_{+}, \mathcal{O}\left(E_{+}\right)\right) \oplus H^{p}\left(M_{-}, \mathcal{O}\left(E_{-}\right)\right) \\
& \rightarrow H^{p}\left(M_{\text {red }}, \mathcal{O}\left(E_{\text {red }}\right)\right) \rightarrow H^{p+1}\left(M_{\text {cut }}, \mathcal{O}\left(E_{\text {cut }}\right)\right) \rightarrow \cdots
\end{aligned}
$$

We think about $M_{c u t}$ as being glued from $M_{ \pm}$along $M_{r e d}$. So we refer to (2.5) as MayerVietoris-type sequence.

Remark 2.10. Wu and Zhang [12, Remark 4.10] conjectured that, if $E$ is a pre-quantum line bundle, then (for a proper choice of the moment map) the cohomology $H^{p}(M, \mathcal{O}(E)$ ) may be calculated by a Mayer-Vietoris-type exact sequence of type (2.5)). In our terms, that would mean that, in this case, $H^{p}(M, \mathcal{O}(E))$ is isomorphic to $H^{p}\left(M_{\text {cut }}, \mathcal{O}\left(E_{\text {cut }}\right)\right)$. 
The long exact sequence (2.5) leads to the following Morse-type inequalities

$$
\begin{aligned}
\sum_{p=0}^{n} t^{p} \operatorname{char} & H^{p}\left(M_{+}, \mathcal{O}\left(E_{+}\right)\right) \\
& +\sum_{p=0}^{n} t^{p} \operatorname{char} H^{p}\left(M_{-}, \mathcal{O}\left(E_{-}\right)\right)+\sum_{p=0}^{n-1} t^{p+1} \operatorname{dim} H^{p}\left(M_{\text {red }}, \mathcal{O}\left(E_{\text {red }}\right)\right) \\
& =\sum_{p=0}^{n} t^{p} \operatorname{char} H^{p}\left(M_{\text {cut }}, \mathcal{O}\left(E_{\text {cut }}\right)\right)+(1+t) Q(\theta, t)
\end{aligned}
$$

for some $Q(\theta, t) \geq 0$. Combining with Theorem 2.7 we obtain the following estimate on $H^{*}(M, \mathcal{O}(E))$ in terms of the cohomology of $M_{ \pm}$and $M_{\text {red }}$

Theorem 2.11. There exists a polynomial $Q^{\prime}(\theta, t) \in \mathcal{L}[t]$, such that $Q \geq 0$ and

$$
\begin{aligned}
& \sum_{p=0}^{n} t^{p} \operatorname{char} H^{p}\left(M_{+}, \mathcal{O}\left(E_{+}\right)\right)+\sum_{p=0}^{n} t^{p} \operatorname{char} H^{p}\left(M_{-}, \mathcal{O}\left(E_{-}\right)\right) \\
& +\sum_{p=0}^{n-1} t^{p+1} \operatorname{dim} H^{p}\left(M_{\text {red }}, \mathcal{O}\left(E_{\text {red }}\right)\right)=\sum_{p=0}^{n} t^{p} \operatorname{char} H^{p}(M, \mathcal{O}(E))+(1+t) Q^{\prime}(\theta, t)
\end{aligned}
$$

In the case when $E$ is a pre-quantum line bundle Theorem 2.11 was conjectured by Wu and Zhang [12, Remark 4.10].

Remark 2.12. 1. The inequalities (2.6) a far from being exact. Hence, Theorem 2.7 together with the Mayer-Vietoris sequence (2.5) give much more information about the cohomology $H^{*}(M, \mathcal{O}(E))$ than Theorem 2.11.

2. The simplest consequence of Theorem 2.11 is the inequalities

$$
\begin{aligned}
& \operatorname{char} H^{0}\left(M_{+}, \mathcal{O}\left(E_{+}\right)\right)+\operatorname{char} H^{0}\left(M_{-}, \mathcal{O}\left(E_{-}\right)\right) \geq \operatorname{char} H^{0}(M, \mathcal{O}(E)) \\
& \operatorname{char} H^{p}\left(M_{+}, \mathcal{O}\left(E_{+}\right)\right)+\operatorname{char} H^{p}\left(M_{-}, \mathcal{O}\left(E_{-}\right)\right)+\operatorname{dim} H^{p-1}\left(M_{\text {red }}, \mathcal{O}\left(E_{\text {red }}\right)\right) \\
& \geq \operatorname{char} H^{p}(M, \mathcal{O}(E)), \quad \text { for any } \quad p=1, \ldots, n .
\end{aligned}
$$

3. Substituting $t=-1$ into (2.7) and using (2.4), we get the gluing formula (2.2). So we obtain a new proof of the gluing formula for Kähler manifolds, which is based on the geometric construction described in Section 3. Note that the standard proof of the gluing formula, [3, 9, 2], uses the Atiyah-Segal-Singer equivariant index theorem. Dietmar Salamon pointed out that the gluing formula for general symplectic manifold can also be proved using our geometric construction by a method similar to [8, Appendix A]. 
Remark 2.13. In the situation considered in this paper many other Morse-type inequalities may be obtained (cf. [12, 10, 1]). It would be very interesting to compare those inequalities.

2.14. Example. We finish this section with a very simple but typical example illustrating Theorem 2.11.

Let $M=\mathbb{C} P^{1}$. We identify $M$ with the 2-dimensional sphere $S^{2} \subset \mathbb{R}^{3}$ and we let $S^{1}$ act on $M$ by rotations around the $z$-axis. This action has two fixed points $P$ and $Q$ (the poles of the sphere). We normalize the Kähler structure on $M$ and the moment map $\mu$ so that $\mu(P)=1, \mu(Q)=-1$. Then the image of $\mu$ is the interval $[-1,1]$ and all the internal points of this interval are regular values of $\mu$.

Let $E$ be an equivariant line bundle over $M$. Then $S^{1}$ acts on the fibers of this bundle over the fixed points $P$ and $Q$. Denote by $r_{Q}, r_{P}$ the weights of these actions. It is well known that $E$ is defined up to an equivariant isomorphism by these weights. In particular (cf., for example, [11, p. 330]) the character of the representation of $S^{1}$ on the cohomology $H^{p}(M, \mathcal{O}(E))$ is given by

$$
\begin{aligned}
& \operatorname{char} H^{0}(M, \mathcal{O}(E))= \begin{cases}\sum_{m=r_{Q}}^{r_{P}} e^{-i m \theta}, & \text { if } \quad r_{Q} \leq r_{P} ; \\
0, & \text { if } \quad r_{Q}>r_{P} ;\end{cases} \\
& \operatorname{char} H^{1}(M, \mathcal{O}(E))=\left\{\begin{array}{lll}
0, & \text { if } \quad r_{Q} \leq r_{P} \\
\sum_{m=r_{P}-1}^{r_{Q}-1} e^{-i m \theta}, & \text { if } \quad r_{Q}>r_{P} .
\end{array}\right.
\end{aligned}
$$

These formulas allow us to calculate the right hand side of (2.7). Let us calculate the left hand side of (2.7).

Since, $M_{\text {red }}$ is a point, $\operatorname{dim} H^{0}\left(M_{\text {red }}, \mathcal{O}\left(E_{\text {red }}\right)\right)=1$ and $\operatorname{dim} H^{1}\left(M_{\text {red }}, \mathcal{O}\left(E_{\text {red }}\right)\right)=0$.

Both manifolds $M_{+}$and $M_{-}$are isomorphic to $\mathbb{C} P^{1}$. The weight of the fiber of the bundle $E_{+}$over $P$ is still equal to $r_{P}$ while the weight of the fiber over $M_{\text {red }}$ is equal to zero. Similarly, the weight of the fiber of the bundle $E_{-}$over $Q$ is equal to $r_{Q}$ while the weight of the fiber over $M_{\text {red }}$ is equal to zero.

Using (2.9), one can now verify Theorem 2.11 in this simple case. For example, if $r_{Q}=r_{P}=r>0$ (this corresponds to the trivial bundle $E=M \times \mathbb{C}$ with a nontrivial circle action), then the left hand side of (2.7) is equal to

$$
\sum_{m=0}^{r} e^{-i m \theta}+t \sum_{m=0}^{r-1} e^{-i m \theta}
$$

\footnotetext{
${ }^{1}$ Note that our signs in the definition of weights and characters (cf. Section 2.1) are different from 11. but agree with [12, 2, 1].
} 
while

$$
\sum_{p=0}^{n} t^{p} \operatorname{char} H^{p}(M, \mathcal{O}(E))=e^{-i r \theta} .
$$

It follows that in this case the polynomial $Q^{\prime}$ of Theorem 2.11 does not depend on $t$ and equals to $\sum_{m=0}^{r-1} e^{-i m \theta}$.

Note also, that the inequalities (2.7), (2.8) become equalities if and only if $r_{Q} \leq 0$ and $r_{P} \geq 0$. This verifies the conjecture of Wu and Zhang [12] (cf. Remark 2.10) in the case $M=\mathbb{C} P^{1}$.

\section{The GeOMETRIC CONSTRUCTION}

In this section we present a geometric construction of a complex manifolds $\Phi$ and a holomorphic map $p: \Phi \rightarrow \mathbb{C}$ such that $p^{-1}(t)$ is isomorphic to $M$ for $t \neq 0$ while $p^{-1}(0)=M_{\text {cut }}$.

3.1. The idea of the construction is the same as in Section 2.3. In fact, we just combine the constructions of $M_{+}$and $M_{-}$together and consider the diagonal action of $S^{1}$ on $M \times \mathbb{C}_{+} \times \mathbb{C}_{-}$. The moment map for this action is given by

$$
\widetilde{\mu}\left(x, z_{+}, z_{-}\right)=\mu(x)-\frac{1}{2}\left|z_{+}\right|^{2}+\frac{1}{2}\left|z_{-}\right|^{2}, \quad x \in M, z_{ \pm} \in \mathbb{C}_{ \pm} .
$$

Zero is a regular value of $\widetilde{\mu}$ and we define $\Phi=\widetilde{\mu}^{-1}(0) / S^{1}$ to be the symplectic reduction of $M \times \mathbb{C}_{+} \times \mathbb{C}_{-}$at zero level.

Clearly, the map $\tilde{p}: M \times \mathbb{C}_{+} \times \mathbb{C}_{-} \rightarrow \mathbb{C}$ defined by the formula

$$
\widetilde{p}:\left(x, z_{+}, z_{-}\right) \mapsto z_{+} z_{-}, \quad x \in M, z_{ \pm} \in \mathbb{C}_{ \pm} .
$$

is $S^{1}$ invariant and, hence, descends to a map $p: \Phi \rightarrow \mathbb{C}$. We think about $\Phi$ as family of complex manifolds $M_{t}=p^{-1}(t)$ parameterized by a complex parameter $t \in \mathbb{C}$.

We endow $\Phi$ with an $S^{1}$-action induced by the action of the circle on the first factor of $M \times \mathbb{C}_{+} \times \mathbb{C}_{-}$. Note that this action preserves the fibers $M_{t}=p^{-1}(t)$ of $p$. Thus $M_{t}(t \in \mathbb{C})$ are also endowed with a holomorphic circle action.

As in Section 2.3 the bundle $E$ induces a bundle $\widetilde{E}$ over $\Phi$. We denote the restriction of $\widetilde{E}$ on $M_{t}$ by $E_{t}$.

The following lemmas describe the fibers of the projection $p$.

Lemma 3.2. For any $t \neq 0$, the fiber $M_{t}=p^{-1}(t)$ is a smooth manifold which is equivariantly symplectomorphic to $M$.

Proof. Fix a nonzero number $t \in \mathbb{C}$. For any $x \in M$ set

$$
r(x)=\sqrt{\mu(x)+\sqrt{\mu(x)^{2}+|t|^{2}}}
$$


and define an embedding

$$
i_{t}: M \rightarrow \widetilde{\mu}^{-1}(0) \cap \widetilde{p}^{-1}(t) \subset M \times \mathbb{C}_{+} \times \mathbb{C}_{-}, \quad i_{t}: x \mapsto\left(x, r(x), \frac{t}{r(x)}\right) .
$$

Clearly, the composition $q \circ i_{t}$ of the above embedding with the natural projection $q$ : $\widetilde{\mu}^{-1}(0) \rightarrow \Phi$ is an equivariant diffeomorphism $M \rightarrow M_{t}=p^{-1}(t)$. Since the map $i_{t}: M \rightarrow$ $M \times \mathbb{C}_{+} \times \mathbb{C}_{-}$is symplectic, so is the composition $q \circ i_{t}$.

Lemma 3.3. For any $t \neq 0$, the fiber $M_{t}=p^{-1}(t)$ is a smooth Kähler manifold which is equivariantly complex isomorphic to $M$. In other words, there exists an equivariant biholomorphic map $\phi_{t}: M \rightarrow M_{t}$.

The pullback $\phi_{t}^{*} E_{t}$ of the bundle $E_{t}=\left.\widetilde{E}\right|_{M_{t}}$ is equivariantly isomorphic to $E$.

Remark 3.4. The manifolds $M$ and $M_{t}$ are not isomorphic as Kähler manifolds. In particular, the map $\phi_{t}$ of Lemma 3.3 is different from the symplectomorphism of Lemma 3.2 .

Proof. Recall from Section 2.2 that the action of the circle group on $M$ extends canonically to a holomorphic action of $\mathbb{C}^{*}$ and consider the diagonal action of $\mathbb{C}^{*}$ on $M \times \mathbb{C}_{+} \times \mathbb{C}_{-}$ (here $z \in \mathbb{C}^{*}$ acts on the second factor by multiplication by $z$ and on the third factor by multiplication by $1 / z)$.

Let $U \subset M \times \mathbb{C}_{+} \times \mathbb{C}_{-}$denote the set of stable points for this action (cf. Section 2.2). Then $\widetilde{p}^{-1}(t) \subset U$ for any $t \neq 0$. Indeed, if $v \in \widetilde{p}^{-1}(t)$ and if the absolute value of the number $z \in \mathbb{C}^{*}$ is large enough, then $\widetilde{\mu}(z \cdot v)<0$, while $\widetilde{\mu}\left(\frac{1}{z} \cdot v\right)>0$. Hence, one can find $z^{\prime} \in \mathbb{C}^{*}$ such that $z^{\prime} \in \widetilde{\mu}^{-1}(0)$.

Fix $t \neq 0$ and consider the complex map

$$
j_{t}: M \rightarrow M \times \mathbb{C}_{+} \times \mathbb{C}_{-}, \quad j_{t}: x \mapsto(x, 1, t) .
$$

Clearly, the image of $j_{t}$ belongs to $\widetilde{p}^{-1}(t)$ and, by the previous paragraph, it belongs also to the set $U$ of stable points for $\mathbb{C}^{*}$ action. Hence, the composition of $j_{t}$ with the quotient $\operatorname{map} q: U \rightarrow U / \mathbb{C}^{*}$ defines an equivariant holomorphic map $\phi_{t}: M \rightarrow M_{t}$. Clearly, $\phi_{t}$ is injective. We claim that $\phi_{t}$ is an isomorphism. By Lemma 3.2, it suffice to show that the image of $j_{t}$ contains the image of the map (3.1). But this follows from the obvious inclusion $\frac{1}{r} \cdot\left(x, r, \frac{t}{r}\right) \in \operatorname{Im}\left(j_{t}\right)$. The first statement of the lemma is proven.

Consider the commutative diagram

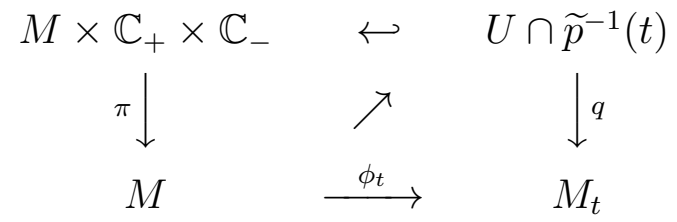


By definition of the bundle $E_{t}$ we have $q^{*} E_{t}=\left.\pi^{*} E\right|_{U \cap \widetilde{p}^{-1}(t)}$. Hence, using $\phi_{t}=q \circ j_{t}$ and $\pi \circ j_{t}=i d$, we obtain

$$
\phi_{t}^{*} E_{t}=j_{t}^{*} q^{*} E_{t}=\phi_{t}^{*} \pi^{*} E=E
$$

Lemma 3.5. The fiber $p^{-1}(0)$ is equivariantly complex isomorphic to the space $M_{\text {cut }}$. If we identify $p^{-1}(0)$ with $M_{\text {cut }}$ using this isomorphism then the restriction $E_{0}$ of $\widetilde{E}$ to $p^{-1}(0)$ is isomorphic to $E_{\text {cut }}$.

Proof. The lemma is an obvious consequence of the equality

$$
\widetilde{p}^{-1}(0)=\left(M \times \mathbb{C}_{+} \times\{0\}\right) \cup\left(M \times\{0\} \times \mathbb{C}_{-}\right) .
$$

For us the most important is the following consequence of the above lemmas

Corollary 3.6. The cohomology $H^{*}\left(M_{t}, \mathcal{O}\left(E_{t}\right)\right)$ of the sheaf of holomorphic sections of the bundle $E_{t}$ is equivariantly isomorphic to $H^{*}(M, \mathcal{O}(E))$ if $t \neq 0$ and is equivariantly isomorphic to $H^{*}\left(M_{\text {cut }}, \mathcal{O}\left(E_{\text {cut }}\right)\right)$ if $t=0$.

\section{Flat morphisms. Proof of Theorem 2.7}

We are in a position now to prove Theorem 2.7. The proof is based on the properties of flat morphisms in complex analysis.

4.1. Flat morphisms. First, we recall some basic facts about flat morphisms. For the details we refer the reader to [5, Sections II.2,III.4].

If $X$ is a complex space and $x \in X$ we denote by $\mathcal{O}(X)$ the sheaf of holomorphic functions on $X$ and by $\mathcal{O}_{x}(X)$ the ring of germs of holomorphic functions at $x$. Let $f: X \rightarrow Y$ be a holomorphic map of complex spaces. For any $y \in Y$, we denote by $X_{y}=f^{-1}(y)$ the fiber of $f$ over $y$.

A holomorphic map $f: X \rightarrow Y$ is called flat at a point $x \in X$ if $\mathcal{O}_{x}(X)$ is a flat module over $\mathcal{O}_{f(x)}(Y)$. Here $\mathcal{O}_{x}(X)$ is considered as $\mathcal{O}_{f(x)}(Y)$-module via the canonical map $f^{*}: \mathcal{O}_{f(x)}(Y) \rightarrow \mathcal{O}_{x}(X)$. The map $f$ is called flat if it is flat at any point $x \in X$.

Let $f: X \rightarrow Y$ be a morphism of complex spaces and suppose $V$ is a holomorphic vector bundle over $X$. For any $y \in Y$, let $V_{y}=\left.V\right|_{X_{y}}$ denote the restriction of $V$ on the fiber $X_{y}$ and let $\mathcal{O}\left(V_{y}\right)$ denote the locally free sheaf of holomorphic sections of $V_{y}$. If $f$ is a flat morphism, then, for any $y \in Y$ and for any pint $\eta \in Y$ closed to $y$, there exists a polynomial $Q(t)$ with nonnegative integer coefficients, such that

$$
\sum_{p} t^{p} \operatorname{dim} H^{p}\left(X_{y}, \mathcal{O}\left(V_{y}\right)\right)=\sum_{p} t^{p} \operatorname{dim} H^{p}\left(X_{\eta}, \mathcal{O}\left(V_{\eta}\right)\right)+(1+t) Q(t) .
$$


The equation (4.1) implies, in particular, that the function $y \mapsto \operatorname{dim} H^{p}\left(X_{y}, \mathcal{O}\left(V_{y}\right)\right)$ is upper semi-continuous, while the holomorphic index of the fibers

$$
\operatorname{ind}\left(V_{y}\right)=\sum_{p}(-1)^{p} \operatorname{dim} H^{p}\left(X_{y}, \mathcal{O}\left(V_{y}\right)\right)
$$

is locally constant on $Y$.

Suppose that in the situation described above a compact Lie group $G$ acts holomorphically on $X$ and $Y$ and that this action commutes with $f$. If the vector bundle $E$ is equivariant with respect to this action then $G$ acts on the cohomology of the fibers. Let char $H^{p}\left(X_{y}, \mathcal{O}\left(V_{y}\right)\right), y \in Y$ denote the character of this action. Then, for any point $\eta \in Y$ closed enough to $y$, there exists a polynomial $Q(t, \theta) \in \mathcal{L}[t]$ (cf. Definition 2.6) such that $Q \geq 0$ and

$$
\sum_{p} t^{p} \operatorname{char} H^{p}\left(X_{y}, \mathcal{O}\left(V_{y}\right)\right)=\sum_{p} t^{p} \operatorname{char} H^{p}\left(X_{\eta}, \mathcal{O}\left(V_{\eta}\right)\right)+(1+t) Q(t, \theta) .
$$

4.2. Proof of Theorem 2.7. It follows now from (4.2) and Corollary 3.6, that in order to prove Theorem 2.7 it suffices to show that the projection $p: \Phi \rightarrow \mathbb{C}$ is flat. But, by a theorem of Kaup and Kerner, [5, Ch. II, Theorem 2.13], any open holomorphic map of smooth complex manifolds is flat. Since, $p$ is open the theorem is proven.

\section{REFERENCES}

[1] M. Braverman, Holomorphic Morse inequalities and symplectic reduction, To appear in Topology.

[2] A. Canas da Silva, Y. Karshon, and S. Tolman, Quantisation of presymplectic manifolds and circle actions, Preprint, dg-ga/9705008, February 1997.

[3] H. Duistermaat, V. Guillemin, E. Meinrenken, and S. Wu, Symplectic reduction and Riemann-Roch for circle actions, Math. Res. Lett. 2 (1995), 259-266.

[4] T. Frankel, Fixed points on Kähler manifolds, Ann. Math. 70 (1959), 1-8.

[5] H. Grauert, Th. Peternell, and R. Remmert, Several complex variables VII, Encyclopaedia of Mathematical Sciences, vol. 74, Springer-Verlag, Berlin Heidelberg New York, 1994.

[6] V. Guillemin and S. Sternberg, Geometric quantization and multiplicities of group representations, Invent. Math 67 (1982), 515-538.

[7] E. Lerman, Symplectic cuts, Math. Res. Lett. 2 (1995), 247-258.

[8] D. McDuff and D. Salamon, J-holomorphic curves and quantum cohomology, University Lecture Series, vol. 6, AMS, Providence, RI, 1994.

[9] E. Meinrenken, Symplectic surgery and the $\operatorname{spin}^{c}$-Dirac operator, To appear in Adv. in Math.

[10] Y. Tian and W. Zhang, Symplectic reduction and analytic localization, Preprint, July 1996.

[11] E. Witten, Holomorphic Morse inequalities, Algebraic and differential topology, Grund. Math. Wiss. 188, Teubner-Texte Math., 70, ed. G. Rassias, Teubner, Leipzig, 1984, pp. 318-333.

[12] S. Wu and W. Zhang, Equivariant holomorphic Morse inequalities III: Non-Isolated fixed points, Adelaide IGA preprint 1997-01; dg-ga/9701009, 1996. 
Institute of Mathematics, The Hebrew University, Jerusalem 91904, IsRael

E-mail address: maxim@math.huji.edu 